\title{
Surface chemistry of first wall materials — from fundamental data to modeling
}

\author{
Ch. Linsmeier ${ }^{\mathrm{a}, *}$, M. Reinelt ${ }^{\mathrm{a}}, \mathrm{K}$. Schmid $^{\mathrm{a}}$ \\ ${ }^{a}$ Max-Planck-Institut für Plasmaphysik, EURATOM Association, \\ Boltzmannstr. 2, 85748 Garching b. München, Germany
}

\begin{abstract}
The application of different materials at the first wall of fusion devices, like beryllium, carbon, and tungsten in the case of ITER, unavoidably leads to the formation of compounds. These compounds are created dynamically during operation and depend on the local parameters like surface temperature, incoming particle energies and species. In dedicated, well-defined laboratory experiments, using mainly X-ray photoelectron spectroscopy and Rutherford backscattering analysis for qualitative and quantitative chemical surface analysis, the parameter space in relevant element combinations are investigated. These studies lead to a deep understanding of the reaction mechanisms under the applied conditions and to a quantitative description of reaction and diffusion processes. These data can be parameterized and integrated into a modeling approach which combines dynamic surface chemistry with the modeling of the transport in the plasma. Two different approaches for surface reaction modeling are compared and benchmarked with experimental data.
\end{abstract}

Keywords:

JNM: Plasma-Materials Interaction, Surface Effects, Chemical Reactions, Diffusion, Alloys and Compounds

PSI-19: Ion-surface interactions, Diffusion, Surface analysis, Beryllium, Tungsten

PACS: 52.40.Hf, 68.35.Fx, 89.30.Jj, 82.65.+r

\footnotetext{
${ }^{*}$ Corresponding author

Email address: linsmeier@ipp.mpg.de (Ch. Linsmeier)
} 


\section{Introduction}

Fusion devices, and in particular all next-step machines, cannot rely on a single material facing the plasma at the first wall. Due to the very different particle and energy loads at different positions within the devices, suitable material solutions are required. The research in plasma-wall interactions over many years finally lead to the choice of beryllium, carbon, and tungsten as the plasma-facing materials for the start-up phase of ITER [1,2]. For the reactor prototypes beyond ITER and also devices beyond this DEMO, the first wall material will most certainly be a tungsten-based material. From the present state of knowledge, tungsten alloys will be the materials of choice. These alloys will be optimized in composition either for safety issues like self-passivation capabilities in case of loss-of-coolant events, and have to be optimized in their thermochechanical properties.

In all cases, material erosion, transport, and re-deposition under operating conditions will lead to surface layers of one material on the other, or more general: to the formation of multi-component surfaces ("mixed materials"). Thermally and ion-driven processes (diffusion, reaction, ion beam mixing) initiate the formation of mixtures and/or compounds of the available elements. In addition, plasma impurities (e.g. oxygen from oxidized surfaces or leaks, nitrogen from seeding of the plasma edge) and the hydrogen isotopes from the fuel can additionally take part in surface chemical processes. The result of all these processes is a first wall surface which dynamically establishes its composition during plasma operation. All chemical and physical erosion processes, the hydrogen isotope inventory determined by hydrogen retention and release, and several thermomechanical properties are influenced by the first wall composition. It is therefore essential to understand the underlying formation and erosion processes in order to be able to predict the first wall surface composition for the different locations within a fusion device.

These processes can be studied in well-defined conditions in dedicated laboratory experiments which allow to specifically vary surface temperatures, surface compositions, particle loads, etc. Dedicated analysis techniques like X-ray photoelectron spectroscopy (XPS) for qualitative and quantitative surface chemistry characterization, Rutherford backscattering spectrometry (RBS) for quantitative surface compositional analysis, and temperature-programmed desorption spectroscopy (TPD) for the analysis of the hydrogen retention and release, allow to characterize the surface conditions under varying plasma exposure scenarios. A number of such studies have been performed in recent years, in particular for the material combinations 
relevant for ITER. Section 2 summarizes these results and shows two examples for thermally and ion-driven processes, respectively. In well-defined experiments reaction mechanisms can be determined, as well as energetic and kinetic parameters for a number of surface reactions. By isolating single processes, a detailed description is possible. Starting from binary reaction systems, the complexity of possible surface reactions increases dramatically when number of components grows: ternary systems, reactive systems involving hydrogen isotopes.

Finally, only a modeling approach based on these surface reaction results and available literature data allows predictions for a complex fusion machine. If the material formation, erosion and hydrogen inventory processes are to be simulated, facilitating predictions for the material behavior e.g. in ITER, for the very different conditions at the first wall locations, it is necessary to reduce the experimental parameter space to few variables. In singling out specific processes in one study, it is possible to determine the parameters which govern temperature or ion-driven processes. By examining the behavior of materials under well-defined conditions, predictions are possible for more complex scenarios. Surface chemistry, nevertheless, is only one aspect in the complex framework which is necessary to simulate the interactions between a fusion plasma and the first wall. Codes treating the fusion plasma itself, the transport of impurities with the respective fluxes towards the wall and reverse, as well as the physics in the plasma edge (like DIVIMP [3], SolPS [4, 5]), are of high complexity and require huge computational power. Adding the surface chemical processes to the overall simulation requires these processes to be treated mathematically and computationally as simple as possible. A sophisticated atomistic treatment of surface chemistry, e.g. by density functional theory, molecular dynamics, or even kinetic Monte Carlo methods, which are from the chemistry point of view very detailed, is excluded for the sake of computational power requirements. Moreover, a benchmarking of these computational techniques with experiments is a tedious and scientifically demanding effort by itself. The predictive power of such calculations is high for a detailed atomistic problem, but due to the complexity these atomistic simulations are quickly at their (computational) limits if the number of parameters, atoms in a calculation cell etc., increases only marginally.

For an integration of surface chemistry into a full simulation of a fusion plasma, we therefore choose a different approach. We use the well-defined surface reaction experiments and the information which can be extracted from the above-mentioned techniques: mainly XPS and RBS. From these techniques, quantitative data e.g. on diffusion constants and energy barri- 
ers for reactions can be determined. Qualitatively, the surface chemistry at different temperatures and particle fluences is described. XPS measurements in binary systems then can be parameterized and these parameters can be benchmarked, e.g. against the well-defined binary reaction experiments. This is demonstrated in section 3 for two different approaches: A homogeneous reaction layer model and a reaction front model. The such determined parameters used for rate equations describing the surface chemistry in multi-component systems can then be integrated into a simulation which includes both the plasma transport and the surface chemistry. In [6] the homogeneous reaction layer model based on the work in [7] is presented which describes the local erosion/deposition processes to occur in a reaction zone of constant thickness and homogeneous composition. This reaction zone exchanges material with the plasma due to erosion/deposition and with an infinite bulk to maintain constant thickness during erosion/deposition. This model can easily be extended to handle reactions beyond erosion/deposition, e.g. chemical reactions and phase formation. This is finally demonstrated in a separate publication in these proceedings [8] for JET, where the surface processes are included.

\section{Surface reaction experiments}

The investigation of the influence of reactions in multi-component surfaces requires experimental conditions in which suitable samples can be produced, characterized, implanted and analyzed under well-controlled environments. Sample preparation involves in a first step the removal of impurity species from the surface and surface-near bulk. The topic of mixed wall materials involves in particular beryllium and carbon. Be is highly reactive (in particular with oxygen and water), and carbon is an ubiquitous contamination. Controlled surface science experiments therefore require base pressures in the low $10^{-10}$, better in the low $10^{-11}$ mbar regime. Furthermore, the performed dedicated experiments involve sample preparation steps where species are deposited from the vapor phase, implanted as energetic ions, or, in the case of oxygen, deposited as atomic species. Some of the experiments involving tungsten surfaces include temperature treatments ranging from $300 \mathrm{~K}$ to $1700 \mathrm{~K}$.

For chemical state analysis, X-ray photoelectron spectroscopy (XPS) is the technique of choice. This method allows for chemical analysis of the surface and surface-near depths both qualitatively (resolving elements and their chemical states) and quantitatively (taking into account models for the depth distribution). XPS is used both in laboratory experiments and 
with synchrotron radiation. The latter case not only enables high resolution spectroscopy, but is in particular useful for gaining chemical information with tunable information depth. This is illustrated below for oxygen ions implanted into a $\mathrm{Be}_{12} \mathrm{~W}$ alloy surface. Thermally driven reactions are investigated in layered systems where one component is the target material and additional components are deposited as layers in the monolayer (ML) to $\mathrm{nm}$ thickness range from the vapor phase. This morphology allows to characterize by XPS the upper layers of the substrate, the interface between the layer and the substrate, and the very surface of the sample. Therefore, reaction and diffusion processes during temperature-driven reactions can be observed.

With respect to the investigation of compound formation processes at the surface of first wall materials, the binary carbon-metal systems are of fundamental interest. Carbon is eroded both by physical and chemical processes in a fusion device. Before more complicated processes at the first wall are investigated (like the influence of compound layers on the hydrogen retention and release), the fundamental interaction processes between carbon and metal surfaces leading to the formation of carbon-based compounds need to be understood.

In general, the interaction of elemental carbon with substrates at different temperatures is of concern, since the wall temperature in a fusion device can range from approximately room temperature to well above $1000 \mathrm{~K}$ during operation. In specific locations where the particle and energy fluxes are very high, the surface temperature may even exceed these values. Reactions between carbon and substrates can also be stimulated by the kinetic energy of particles escaping the plasma and reaching the first wall. The investigations on binary carbon-metal systems, both for thermally and ion-induced reactions, are summarized with respect to certain aspects and materials in $[9-12]$.

The interaction of carbon with beryllium and tungsten is of specific interest due to the application of these elements as first wall materials in ITER. The initial stages of carbide formation on beryllium and carbon diffusion into the substrate have been investigated with carbon films deposited on Be single crystals $[9,13,14]$. Reactive processes of C-containing ions with beryllium are studied by $\mathrm{C}^{+}$and $\mathrm{CO}^{+}$implantation $[15,16]$. The concentration-dependent diffusion coefficient of Be in graphite and the influence of oxygen at the interface is studied in a combined XPS and RBS depth profile analysis [17]. Although earlier work is available on the $\mathrm{C}-\mathrm{Be}$ interaction [18-24], the system was studied for the first time without any additionally present contaminations, in particular $\mathrm{BeO}$ (in some cases even 
exceeding the amount of measured beryllium carbide in earlier work).

The interaction of carbon films with tungsten surfaces is investigated thermally both for polycrystalline $\mathrm{W}[25,10]$ and $\mathrm{W}(111)$ surfaces, as well as for $\mathrm{Ar}^{+}$implantation [12]. As an example, the reactions of $\mathrm{C}$ on W(111) are shown in Fig. 1 for a $3.9 \mathrm{~nm} \mathrm{C}$ film on W(111). As a function of annealing temperature, four different regions can be identified. During phase (I), no additional carbide is formed and only the transition of disordered graphitic $\mathrm{C}$ into graphitic $\mathrm{C}$ is observed, the peak intensity of disordered $\mathrm{C}$ (at $284.5 \mathrm{eV}$ in [25]) decreases with annealing temperature. In phase (II), the diffusion of carbon into the $\mathrm{W}$ substrate starts. This phase starts on $\mathrm{W}(111)$ around $1000 \mathrm{~K}$, whereas on polycrystalline $\mathrm{W}$ the onset is approximately $100 \mathrm{~K}$ lower. During phase (II), also the formation of tungsten subcarbide, $\mathrm{W}_{2} \mathrm{C}$, starts. The $\mathrm{C} 1 s$ binding energy for this compound (and also for WC) is determined in separate experiments in which $100 \mathrm{~nm}$ tungsten on graphite are annealed to $1070 \mathrm{~K}$ and $1370 \mathrm{~K}$. In subsequent XPS measurements, the binding energies for $\mathrm{W}_{2} \mathrm{C}$ and $\mathrm{WC}$ are determined to 283.6 and $283.1 \mathrm{eV}$, respectively. The corresponding $\mathrm{W} 4 f_{7 / 2}$ binding energies are 31.8 and $32.2 \mathrm{eV}$. With these binding energies, a chemical phase analysis of the $\mathrm{C} 1 s$ signal by peak fitting allows the precise quantitative composition analysis. Phase (III) in the temperature diagram is characterized by a constant $\mathrm{C}$ amount within the XPS analysis depth. $\mathrm{W}_{2} \mathrm{C}$ dominates, although the concentration of WC is slightly increased compared to the phases (I) and (II). In case of polycrystalline $\mathrm{W}$, phase (II) extends approximately between 1000 and $1250 \mathrm{~K}$, whereas on $\mathrm{W}(111)$ the stability phase of $\mathrm{W}_{2} \mathrm{C}$ extends up to $1470 \mathrm{~K}$. In phase (IV) at the highest temperatures carbon diffusion into tungsten accelerates again and finally only $\approx 10 \%$ of the surface within the XPS analysis depth consists of carbon. $\mathrm{W}_{2} \mathrm{C}$ decreases and $\mathrm{WC}$ becomes the dominating carbide compound at highest temperatures.

Reaction kinetic parameters can be determined at several annealing temperatures for carbon on $\mathrm{W}_{\text {poly }}$ and $\mathrm{W}(111)$ substrates for the formation reaction of $\mathrm{W}_{2} \mathrm{C}$. Grain boundary diffusion influences the activation energy for the subcarbide formation on these substrates: Whereas the activation energy for the $\mathrm{W}_{2} \mathrm{C}$ formation on $\mathrm{W}_{\text {poly }}$ is $0.8 \mathrm{eV}$, the corresponding value for $\mathrm{W}(111)$ is $1.1 \mathrm{eV}$. Qualitatively, the influence of the grain boundaries is also visible in the carbon amounts during the phase (III). On the polycrystalline substrate, phase (III) not only exists in a smaller temperature range [25] than on W(111), but also the amount of carbon is not constant over the whole phase (III) duration.

Moreover, iron and nickel as main components in steels are investigated. These metals are of particular scientific interest, since the carbide formation 
reaction for $\mathrm{Fe}$ and $\mathrm{Ni}$ is endothermic under standard conditions [26-28].

Finally, the temperature-dependent reaction and diffusion processes of carbon films on Ti and Si surfaces are studied both since Ti and Si are used in current fusion devices, and as a reference in comparison to the materials above $[29,9]$. Surface chemistry induced by inert ions is investigated for $\mathrm{C}-\mathrm{Ti}$ systems both for $\mathrm{C}$ layers on $\mathrm{Ti}$ and vice versa by $\mathrm{Ar}^{+}$and $\mathrm{He}^{+}$ bombardment at several ion energies [30], as well as for $\mathrm{D}^{+}$ion bombardment [31]. Finally, erosion of $\mathrm{C}$ layers on $\mathrm{Be}, \mathrm{Ti}$, and $\mathrm{Ta}$ substrates was investigated in order to determine the substrate mass effect [32].

The beryllium-tungsten binary system is also of interest due to the ITER material selection. The bulk binary phase diagram predicts the formation of several alloy phases [33]. In several series of experiments [34-37] and theoretical approaches [38, 39], Be-W interaction were studied, as well as in in collaboration with the PISCES group [40]. For Be deposition on W, the system is characterized by a delicate alloying-sublimation balance: A nm thin $\mathrm{Be}_{2} \mathrm{~W}$ layer is formed above $670 \mathrm{~K}$, additional Be sublimates already above $770 \mathrm{~K}$. In the reverse system, W deposited on Be, the formation of a Be-W alloy starts only above $970 \mathrm{~K}$, as determined from RBS spectra. However, this technique is not sensitive enough to exclude the possibility of an interface alloy formation in the order of a monolayer, as observed in the $\mathrm{Be} / \mathrm{W}$ experiments. The stoichiometry of the alloy observed at $T>970 \mathrm{~K}$ is $\mathrm{Be}_{12} \mathrm{~W}$. From the temporal evolution of the alloy layer thickness a diffusion coefficient of $1.6 \cdot 10^{-13} \mathrm{~cm}^{2} \mathrm{~s}^{-1}$ at $1070 \mathrm{~K}$ is determined [36]. For 1023 and $1123 \mathrm{~K}$, additional diffusion coefficients for the $\mathrm{Be}-\mathrm{W}$ interdiffusion are available: $4.3 \cdot 10^{-15} \mathrm{~cm}^{2} \mathrm{~s}^{-1}$ and $5.8 \cdot 10^{-13} \mathrm{~cm}^{2} \mathrm{~s}^{-1}$, respectively [41].

As an example for depth-resolved chemical analysis, Fig. 2 shows photoelectron spectra in the $\mathrm{Be} 1 s$ and $\mathrm{W} 4 f$ regions for a $\mathrm{Be}_{12} \mathrm{~W}$ layer, implanted with oxygen ions at $0.5-1 \mathrm{keV}$, with the substrate at room temperature. The measurements are performed at the synchrotron HZB-BESSY II where the photon energy is tuned for each spectrum such that the kinetic energy of the photoelectrons from both Be $1 s$ and $\mathrm{W} 4 f$ are 125, 225, 475, and $685 \mathrm{eV}$, respectively. As a consequence, the information depths for both spectral regions are identical. A low kinetic photoelectron energy guarantees surface sensitivity. From the peak positions it is obvious that even at room temperature, the $\mathrm{Be}_{12} \mathrm{~W}$ alloy reacts with the oxygen ions and forms several compounds. Close to the surface, Be is oxidized and forms $\mathrm{BeO}$, leaving metallic $\mathrm{W}$ behind. With increasing depth, the formation of ternary $\mathrm{Be}-\mathrm{W}-$ $\mathrm{O}$ compounds becomes more prominent. Both the tungsten bronze $\mathrm{BeWO}_{3}$ and the beryllium tungstate $\mathrm{BeWO}_{4}$ with their characteristic peak shifts are visible, in particular in the $\mathrm{W} 4 f$ region. Neither $\mathrm{WO}_{3}$ nor metallic Be 
appear within the investigated depth zone. Further analysis of this data is still ongoing.

\section{Surface reaction modeling}

\subsection{Simplified reaction front model (RFM)}

As described above, the XPS experiments, as a first approximation, can be treated as one-dimensional layered systems. Solid state reactions between the layers occur therefore mainly via diffusive processes forming a product layer of increasing thickness between two reactant layers. In accordance with this picture, we construct a simplified model based on stacked layers of chemical phases. The term chemical phase is used here to describe a thermodynamically stable material with a constant composition (e.g. Be, $\mathrm{C}, \mathrm{W}, \mathrm{Be}_{2} \mathrm{C}, \mathrm{Be}_{12} \mathrm{~W}$, etc). If we start with a layer of chemical phase $\mathrm{A}$ on top of chemical phase $\mathrm{B}$, a product layer can be formed between $\mathrm{A}$ and $\mathrm{B}$ consisting for example of the chemical phase $A_{x} B_{y}$.

$$
x \mathrm{~A}+y \mathrm{~B} \longrightarrow \mathrm{A}_{\mathrm{x}} \mathrm{B}_{\mathrm{y}}
$$

In the one-dimensional model, the reaction is only possible if a flux of A traverses the product layer $\mathrm{A}_{\mathrm{x}} \mathrm{B}_{\mathrm{y}}$ and reaches $\mathrm{B}$. This flux instantly forms $A_{x} B_{y}$, also consuming $B$ in the process, for a diffusion limited solid state reaction. This assumption is justified if the difference in the activation energies for the local phase formation and the material transport by diffusion is large enough. In such a case, the energetic processes forming the phase by local atomistic rearrangement can be neglected. Of course, a diffusive flux of $\mathrm{B}$ through $\mathrm{A}_{\mathrm{x}} \mathrm{B}_{\mathrm{y}}$ also forms product $\mathrm{A}_{\mathrm{x}} \mathrm{B}_{\mathrm{y}}$ and consumes $\mathrm{A}$ accordingly at the opposite interface. The gradient that drives the diffusion can, as an approximation, be seen as the concentration gradient between the volume density of the pure reactant (A or B) and 0 at the opposite reaction front across the thickness of the product layer. In a diffusion-like approach, the flux of $\mathrm{A}$ across the product layer $\mathrm{A}_{\mathrm{x}} \mathrm{B}_{\mathrm{y}}$ of thickness $\Delta L$ can be given as

$$
\frac{d \rho_{A}}{d t}=D \frac{\Delta \rho_{A}}{\Delta L}
$$

where $\rho_{A}$ [particles $\left./ \mathrm{m}^{3}\right]$ is the volume density of the pure chemical phase A, $t[\mathrm{~s}]$ is time, $D\left[\mathrm{~m}^{2} / \mathrm{s}\right]$ is the diffusivity of $\mathrm{A}$ in $\mathrm{A}_{\mathrm{x}} \mathrm{B}_{\mathrm{y}}$ and is approximated as

$$
D(T)=D_{0} \exp \left(\frac{-\Delta E_{\text {Diff }}}{k T}\right)
$$


and therefore only temperature dependent. It should be noted that the diffusivity is actually also a function of the concentrations

$$
C_{X}=\frac{\sigma_{X}}{\sum \sigma}
$$

i.e. $D\left(T, C_{A}, C_{B}, C_{A B}\right)$ with $\sigma$ [particles $\left./ \mathrm{m}^{2}\right]$ as the areal density (interdiffusivity). This concept is not implemented here.

As long as there is more than $1 \mathrm{ML}$ of reactant left, the volume density difference $\Delta \rho$ is constant, but the increasing thickness of the product layer leads to a square root dependence of the material increase of the product with time at constant temperature. This is to be expected for a typical diffusion-limited solid state reaction. For simplicity, the model assumes a linear decrease of the volume density from the pure chemical phase to 0 for reactant thicknesses smaller than 1 ML. Because the diffusive flux of reactants is directly converted to product formation, there is no concentration build-up of $\mathrm{A}$ in the layer $\mathrm{A}_{\mathrm{x}} \mathrm{B}_{\mathrm{y}}$ included in the model. This also restricts the free parameters of the model to 4 per reactive layer (Diffusivities of $\mathrm{A}$ in $A_{x} B_{y}$ and $B$ in $A_{x} B_{y}$, each described by equ. 3 ).

Dissociation of phases according to

$$
\mathrm{A}_{\mathrm{x}} \mathrm{B}_{\mathrm{y}} \longrightarrow x A+y \mathrm{~B}
$$

are more difficult to include into this picture, because they would require a mixing of different chemical phases within a layer, which is not possible by this model. Therefore, we assume for dissociation of a compound chemical phase $A_{x} B_{y}$ that the resulting material fluxes of products $A$ and $B$ are added to their respective connected layers of $\mathrm{A}$ and $\mathrm{B}$.

In total, phase $\mathrm{A}$ is consumed by the diffusive flux to the interface forming product $\mathrm{A}_{\mathrm{x}} \mathrm{B}_{\mathrm{y}}$ and produced by the parallel occurring dissociation of $\mathrm{A}_{\mathrm{x}} \mathrm{B}_{\mathrm{y}}$.

$$
\frac{d \sigma_{A}}{d t} \approx-\frac{D_{\mathrm{AinAB}} \rho_{A}}{\Delta L_{A B}}+x \sigma_{A B} \nu \exp \left(\frac{-\Delta E_{\text {Diss }}}{k T}\right)
$$

The second term is of a simple first order Arrhenius type containing the dissociation enthalpy $\Delta E_{\text {Diss }}$ and a first order reaction constant $\nu\left(10^{13} \mathrm{~s}^{-1}\right.$, from lattice vibrations is solid state reactions) as an approximation for the activated dissociation of $A_{x} B_{y}$. The total equation for $B$ is equivalent.

The total reaction flux forming $\mathrm{A}_{\mathrm{x}} \mathrm{B}_{\mathrm{y}}$ is therefore composed of the diffusive fluxes $\mathrm{A}$ and $\mathrm{B}$ across the product layer, weighted by the stoichiometry of $\mathrm{A}_{\mathrm{x}} \mathrm{B}_{\mathrm{y}}$ minus the dissociative flux of $\mathrm{A}_{\mathrm{x}} \mathrm{B}_{\mathrm{y}}$ :

$$
\frac{d \sigma_{A B}}{d t} \approx \frac{D_{\mathrm{AinAB}} \rho_{A}}{x \Delta L_{A B}}+\frac{D_{\mathrm{BinAB}} \rho_{B}}{y \Delta L_{A B}}-\sigma_{A B} \nu \exp \left(\frac{-\Delta E_{\text {Diss }}}{k T}\right)
$$


with

$$
\Delta L_{A B}=\frac{\sigma_{A B}}{\rho_{A B}}
$$

The coupled equations 6 and 7 can be solved numerically. In the simplest case, the RFM corresponds to two reactant layers, separated by the layer containing the product. It can be extended to a more complex series of stacked triple layers. This yields a time and temperature dependent evolution of rectangular depth profiles of a set of stacked chemical phases, parameterized by a small number of parameters.

\subsection{Comparison with experimental data}

We use this model to simulate the XPS laboratory experiments described in section 2. However, to be able to directly compare the calculated time dependent series of depth profiles to XPS measurements, we need to take the limited depth information of XPS into account. Therefore we construct artificial XPS spectra with a forward calculation from the rectangular depth profiles. It can be assumed that the photon flux from the X-ray source excites the atoms of the sample homogeneously up to a large depth (approx. micrometer) compared to the escape depth of the photoelectrons. If the near-surface region of the sample is discretized into $n$ layers of thickness $d_{i}$, then the irradiation creates a flux of photoelectrons from an element $\mathrm{X}$ in each layer that is

$$
I_{i}^{0} \propto \frac{P C_{x} S_{x} d_{i}}{\lambda_{i}}
$$

where $P$ is the photon flux, $C_{x}$ is the concentration of element $\mathrm{X}, S_{x}$ is a sensitivity factor for this element as used in [42] and $\lambda_{i}$ is the inelastic mean free path of the photoelectrons within the layer $i$. This flux of photoelectrons starting at layer $i$ in the direction of the surface is attenuated by the layers $j$ between $i$ and the surface. Because each layer $j$ can have a different material composition, its $\lambda_{j}$ can also change accordingly. Therefore, the remaining intensity that reaches the surface (emitted by layer $i$ ) can be modeled as an exponential decay according to

$$
I_{i}=I_{i}^{0} \prod_{j=i}^{0} \exp \frac{-d_{j}}{\lambda_{j} \cos \theta}
$$

with $\theta$ as the angle between analyzer and surface normal. Finally, the total photoelectron flux $I_{\text {Total }}$ emitted from a chemical phase in a sample that 
reaches the surface is then proportional to

$$
I_{\text {Total }} \propto \sum_{i=n}^{0} I_{i}
$$

with intensity contributions from the surface up to a depth of $\sum_{n} d_{j}$. The apparatus function, transmission and element-specific photoionization cross sections are all combined in the empirical sensitivity factor $S_{x}$ [42]. Because the intensity contribution decreases exponentially, a calculation of the contributions up to $50 \mathrm{~nm}$ usually is sufficient for small inelastic mean free paths (IMFP) $\lambda$. To estimate the IMFP we use the semiempirical model for inorganic compounds given in [43] for all included chemical phases. Evaluating only the photoelectron intensity ratios eliminates the unknown (but constant) photon flux $P$. With this approach, the limited information depth and depth-dependent information contribution, which strongly depends on the material properties, is taken into account.

Fig. 3a shows the experimental temperature dependent chemical evolution of a $2.5 \mathrm{~nm}$ Be film deposited onto a polycrystalline $\mathrm{W}$ substrate. In Fig. 3c, the results from the RFM and XPS forward modeling calculation are shown. Several features of the experiment can be reproduced by the simulation. The experiments show the appearance of $\mathrm{Be}_{2} \mathrm{~W}$ and the simultaneous disappearance of Be starting at $700 \mathrm{~K}$. The surface alloy disappears above $1100 \mathrm{~K}$. Because oxidation of Be is observed even under good ultrahigh vacuum conditions, the oxidation of the surface is also included as an additionally coupled reaction tuple. The resulting initial layered structure is therefore adsorbed oxygen $(0 \mathrm{~nm})-\mathrm{BeO}(0.1 \mathrm{~nm})-\mathrm{Be}(2.5 \mathrm{~nm})-\mathrm{Be}_{2} \mathrm{~W}$ $(0 \mathrm{~nm})-\mathrm{W}$ (1 mm, substrate). The activation energy for diffusion of Be in $\mathrm{Be}_{2} \mathrm{~W}$ is set to $\Delta E_{\text {Diff }}=1.5 \mathrm{eV}$ with a diffusion constant $D_{0}=10^{-12} \mathrm{~m}^{2} \mathrm{~s}^{-1}$. $\mathrm{W}$ is not allowed to diffuse into the alloy. The dissociation of $\mathrm{Be}_{2} \mathrm{~W}$ is implemented with $\Delta E_{\text {Diss }}=3.1 \mathrm{eV}$ and $\nu=10^{13} \mathrm{~s}^{-1}$. Dissociation of the surface alloy starting at $1100 \mathrm{~K}$ creates free Be which can sublimate. The sublimation flux of Be into the vacuum included in the model is slightly higher than one would expect from the standard vapor pressure data of pure metallic Be. This can be attributed to the fact that the equilibrium surface concentration of free Be at high temperature is not high enough to allow the formation of an extended metallic phase. Although the model proposed here is based on simple rectangular one-dimensional depth profiles, the XPS results can be parameterized quite well. However, the material transport parameters derived from this model are only rough approximations of the multi-dimensional diffusivity functions. Also, possible deviations from the 
base assumption of a one-dimensional layered structure, like island formation, are not included. Such surface morphology changes can influence the measured results significantly. Therefore, further investigations also need to take possible $2 \mathrm{D}$ or $3 \mathrm{D}$ morphology evolutions into account.

\subsection{Homogeneous mixture model (HMM) based on coupled rate equations}

A still more simplified parametrization of the XPS experiments is used in combination with the global transport code described in $[6,8]$. For plasma irradiated surfaces, the collision cascades induced by the ions change the depth profiles and lead the system away from thermodynamic equilibrium by displacements and energy deposition. These processes are in good approximation independent of the substrate temperature. The thermally induced chemical phase formations and dissociations nevertheless occur parallel to the ion induced modifications, thus rearranging the system back to a thermodynamically favorable state. These processes can be described in a simplified way by Arrhenius type equations.

$$
\begin{aligned}
& y_{1} \mathrm{X}_{1}+y_{2} \mathrm{X}_{2}+y_{3} \mathrm{X}_{3}+\ldots \longrightarrow \mathrm{X}_{\mathrm{n}} \\
& \Gamma_{j}=\left(\prod_{i=1}^{n} \sigma_{X_{i}}{ }^{y_{i}}\right) r \exp \left(-\frac{\Delta E_{j}}{k T}\right)
\end{aligned}
$$

The reaction flux $\Gamma_{j}$ of an elemental reaction depends on the product of the areal densities of all involved chemical phases $\mathrm{X}_{i}$ to the power of their stoichiometric coefficient and the Boltzmann term, containing an activation energy. This activation energy has no direct physical equivalent in solid state reactions (in contrast to liquid reactions) and is a free parameter of the model. Also the reaction constant $r$ is a free parameter. It is set to $10^{13} \mathrm{~s}^{-1}$ for first order reactions and is in the order of $10^{-10} \mathrm{~m}^{2} \mathrm{~s}^{-1}$ and $10^{-30} \mathrm{~m}^{4} \mathrm{~s}^{-1}$ for second and third order reactions, respectively. These parameters can be obtained from the direct parameterization of XPS measurements.

The total reaction flux changing the areal density $\sigma$ of a chemical phase $\mathrm{X}$ at the surface is the sum of all contributing elemental reactions (either forming of destroying the phase), weighted by the stoichiometric coefficient which with $\mathrm{X}$ appears in the respective reaction

$$
\frac{d \sigma_{X_{i}}}{d t}=\sum y_{j} \Gamma_{j}^{\text {Formation }}-\sum y_{j} \Gamma_{j}^{\text {Dissociation }}
$$

This expression represents a set of coupled ordinary differential equations for a set of $i$ chemical phases $\mathrm{X}_{i}$ including all reaction pathways and can be solved numerically.

This approach has several advantages: 
- It is simple enough to be included into a larger modeling context.

- The homogeneous distribution of chemical phases is a good approximation for a surface under high particle flux bombardment, where the collision cascades lead to a strong intermixing of atoms in parallel to the diffusive processes.

- The resulting reaction flux can be coupled to influxes from the plasma into the surface (deposition), destructive fluxes by cascade induced phase dissociation within the surface, and fluxes leaving the surface (sputtering, chemical erosion, sublimation, etc.).

- By coupling of elemental reactions (binary formations and dissociations), complex mixtures, as they occur in multi-element experiments such as ITER, can be approximated even if they are not fully evaluated experimentally.

Fig. 3b and d show the comparison of the HMM and an experiment, where $1.4 \mathrm{~nm}$ of Be were deposited onto a pyrolytic graphite substrate, flash-heated step-wise and investigated by XPS. It can be seen that the agreement is not quantitative, because the zero-dimensional HMM does not include depth profiles as the previously discussed one-dimensional RFM. In particular the areal density of the substrate is an additional arbitrary parameter of the model. The amount of substrate available for the reactions must be artificially restricted to avoid too high reaction rates. Moreover, the continuous increase of the $\mathrm{C}$ fraction, as observed in the experiment and shown in Fig. 3b, is not reproduced in the calculation (Fig. 3c). This increase is attributed to a morphology change during carbide formation which is not included in the HMM. However, the temperatures at which specific phases dominate the surface can be reproduced by the model. The formation and dissociation of $\mathrm{Be}_{2} \mathrm{C}$ start at 600 and $1100 \mathrm{~K}$, respectively. The formation of beryllium carbide $\left(2 \mathrm{Be}+\mathrm{C} \longrightarrow \mathrm{Be}_{2} \mathrm{C}\right)$ is reproduced in the model with an activation energy of $1.8 \mathrm{eV}$. The dissociation of beryllium carbide $\left(\mathrm{Be}_{2} \mathrm{C} \longrightarrow 2 \mathrm{Be}+\mathrm{C}\right.$, assumed enthalpy of dissociation: $\left.3.1 \mathrm{eV}\right)$ allows free $\mathrm{Be}$ to appear at high temperatures and therefore evaporation of $\mathrm{Be}(\mathrm{Be} \longrightarrow \mathrm{Be} \uparrow)$, leaving $\mathrm{C}$ at the surface. The sublimation flux of free Be is derived from the standard vapor pressure data of metallic Be. The oxidation of $\mathrm{Be}\left(\mathrm{Be}+\mathrm{O}_{\text {adsorbed }} \longrightarrow \mathrm{BeO}\right)$ and the dissociation of the oxide $\left(2 \mathrm{BeO} \longrightarrow 2 \mathrm{Be}+\mathrm{O}_{2} \uparrow\right)$ are also coupled to the system and limited by the partial pressure of oxygen in the UHV chamber. An increased pressure of $10^{-8}$ mbar is assumed, which can be explained by the degassing of the sample surrounding during the heating phase in the experiment. The sticking 
coefficient for $\mathrm{O}_{2}$ is set to unity because of the high reactivity of metallic Be. A maximum of $1 \mathrm{ML}\left(10^{19} \mathrm{~m}^{-2}\right)$ of oxygen can be adsorbed on the surface and react with other chemical phases like Be.

\section{Summary}

Dynamic surface compositions at the first wall of fusion devices with more than one element as wall material are a consequence of erosion, transport and re-deposition processes during operation. For ITER with its Be, C, O material mix, but also for next-step DEMO and reactor devices, material mixing will be an unavoidable process. In order to predict the physical, chemical first wall properties, the knowledge of surface chemical processes under the in-vessel conditions is necessary. A number of fundamental surface chemistry studies in well-defined laboratory environments provide insight in reaction mechanisms for the relevant materials as function of temperature, impinging particle energy end species. Based on the binary systems experimentally investigated in particular by XPS and RBS, a set of parameterized reaction steps can be defined. These parameterized reactions in binary systems can then be combined to describe the necessary reaction mix in different simplified modeling approaches. These parameterized reactions can be integrated into a more general model scenario which combines the plasma transport, plasma edge properties, and the respective incoming and outgoing particle fluxes. Such, the dynamical changes under locally different wall conditions can be integrated into a modeling scenario. The coupling of elemental reaction steps, constructs a simplified model for a multi-element surface chemistry description. The here proposed models can help to evaluate complex systems such as the full ITER relevant $\mathrm{Be}-\mathrm{W}-\mathrm{C}-\mathrm{O}-\mathrm{N}(\ldots)$ elemental mix, which is not directly accessible in full detail by laboratory experiments. As next steps, further selected benchmark tests are required to explore the limits of the proposed parameterizations.

\section{Acknowledgement}

We thank F. Kost and M. Köppen for providing unpublished data, and A. Vollmer for the support with the HZB-BESSY II measurement campaigns.

[1] ITER Physics Basis Editors et al., Nucl. Fusion 39 (1999) 2137.

[2] K. Ikeda and et al., Nucl. Fusion 47 (2007).

[3] P.C. Stangeby and J.D. Elder, J. Nucl. Mater. 196-198 (1992) 258 . 
[4] D. Reiter, H. Kever, G.H. Wolf, M. Baelmans, R. Behrisch, and R. Schneider, Plasma Phys. Controlled Fusion 33 (1991) 1579.

[5] R. Schneider, D. Reiter, H.P. Zehrfeld, B. Braams, M. Baelmans, J. Geiger, H. Kastelewicz, J. Neuhauser, and R. Wunderlich, J. Nucl. Mater. 196-198 (1992) 810 .

[6] K. Schmid, these proceedings .

[7] K. Schmid, M.J. Baldwin, R.P. Doerner, and D. Nishijima, Nucl. Technol. 159 (2007) 238.

[8] M. Reinelt, these proceedings .

[9] Ch. Linsmeier, J. Luthin, and P. Goldstraß, J. Nucl. Mater. 290-293 (2001) 25.

[10] Ch. Linsmeier, J. Roth, and K. Schmid, IAEA Atomic and PlasmaMaterial Interaction Data for Fusion 12 (2003) 79.

[11] Ch. Linsmeier, J. Luthin, K.U. Klages, A. Wiltner, and P. Goldstraß, Phys. Scr. T111 (2004) 86.

[12] Ch. Linsmeier, AIP Conf. Proc. 740 (2004) 182.

[13] P. Goldstraß and Ch. Linsmeier, Nucl. Instrum. Methods B 161-163 (2000) 411.

[14] P. Goldstraß, K.U. Klages, and Ch. Linsmeier, J. Nucl. Mater. 290-293 (2001) 76 .

[15] P. Goldstraß, W. Eckstein, and Ch. Linsmeier, J. Nucl. Mater. 266-269 (1999) 581.

[16] P. Goldstraß and Ch. Linsmeier, J. Nucl. Mater. 290-293 (2001) 71.

[17] K. Schmid, A. Wiltner, and Ch. Linsmeier, Nucl. Instrum. Methods B 219 (2004) 947.

[18] T.G. Nieh, J. Wadsworth, and A. Joshi, Scripta Metallurgica 20 (1986) 87.

[19] K. Ashida, K. Watanabe, and T. Okabe, J. Nucl. Mater. 241-243 (1997) 1060. 
[20] K. Ashida and K. Watanabe, Fusion Eng. Des. 37 (1997) 307.

[21] J. Roth, W.R. Wampler, and W. Jacob, J. Nucl. Mater. 250 (1997) 23.

[22] R.A. Anderl, G.R. Longhurst, R.J. Pawelko, and M.A. Oates, J. Fusion Energy 16 (1997) 95.

[23] C.-T. Tzeng, K.-D. Tsuei, and W.-S. Lo, Phys. Rev. B 58 (1998) 6837.

[24] R.A. Anderl, R.A. Causey, J.W. Davis, R.P. Doerner, G. Federici, A.A. Haasz, G.R. Longhurst, W.R. Wampler, and K.L. Wilson, J. Nucl. Mater. 273 (1999) 1.

[25] J. Luthin and Ch. Linsmeier, Surf. Sci. 454-456 (2000) 78.

[26] A. Wiltner and Ch. Linsmeier, Phys. Status Solidi A 201 (2004) 881.

[27] A. Wiltner and Ch. Linsmeier, Surf. Sci. 602 (2008) 3623 .

[28] A. Wiltner, Ch. Linsmeier, and T. Jacob, J. Chem. Phys. 129 (2008) 084704 .

[29] J. Luthin and Ch. Linsmeier, Phys. Scr. T91 (2001) 134.

[30] J. Luthin, H. Plank, J. Roth, and Ch. Linsmeier, Nucl. Instrum. Methods B 182 (2001) 218.

[31] K.U. Klages, A. Wiltner, J. Luthin, and Ch. Linsmeier, J. Nucl. Mater. 313-316 (2003) 56.

[32] M. Reinelt and Ch. Linsmeier, Nucl. Instrum. Meth. Phys. Res. B 258 (2007) 270.

[33] T.B. Massalski, H. Okamoto, P.R. Subramanian, and L. Kacprzak, Binary Alloy Phase Diagrams (ASM International, Materials Park, Ohio, 1996), 2nd ed., version 1.0 edition.

[34] A. Wiltner and Ch. Linsmeier, J. Nucl. Mater. 337-339 (2005) 951.

[35] A. Wiltner and Ch. Linsmeier, New J. Physics 8 (2006) 181.

[36] Ch. Linsmeier, K. Ertl, J. Roth, A. Wiltner, K. Schmid, F. Kost, S.R. Bhattacharyya, M. Baldwin, and R.P. Doerner, J. Nucl. Mater. 363-365 (2007) 1129. 
[37] A. Wiltner, F. Kost, S. Lindig, and Ch. Linsmeier, Phys. Scr. 128 (2007) 133.

[38] A. Allouche and Ch. Linsmeier, J. Phys.: Conf. Ser. 117 (2008) 012002.

[39] A. Allouche, A. Wiltner, and Ch. Linsmeier, J. Phys.: Cond. Matter 21 (2009) 355011.

[40] R.P. Doerner, M. Baldwin, J. Hanna, Ch. Linsmeier, D. Nishijima, R. Pugno, J. Roth, K. Schmid, and A. Wiltner, Phys. Scr. 128 (2007) 115 .

[41] M.J. Baldwin, R.P. Doerner, D. Nishijima, D. Buchenauer, W.M. Clift, R.A. Causey, and K. Schmid, J. Nucl. Mater. 363-365 (2007) 1179.

[42] J.F. Moulder, W.F. Strickle, P.E. Sobol, and K.D. Bomben, Handbook of X-ray Photoelectron Spectroscopy (Ed.: J. Chastain, Perkin-Elmer Corp., Minnesota, 1992).

[43] W.H. Gries, Surf. Interface Anal. 24 (1996) 38. 
Figure 1: Surface composition of a $4 \mathrm{~nm}$ carbon film deposited on a W(111) single crystal, determined from the C $1 s$ XPS spectra measured during an annealing series.

Figure 2: Photoelectron spectra in the $\mathrm{W} 4 f$ and Be $1 s$ binding energy regions, measured with different photon energies after $\mathrm{O}^{+}$implantation into a $\mathrm{Be}_{12} \mathrm{~W}$ alloy layer. The spectra show different chemical compositions from surface (upper spectra) to increasing depths.

Figure 3: Comparison between XPS experimental data of binary layered systems (left column: $2.5 \mathrm{~nm}$ Be on $\mathrm{W}$, right column: $1.4 \mathrm{~nm}$ Be on $\mathrm{C}$ ) and the results of a reaction front (c) and a homogeneous mixture model (d). 


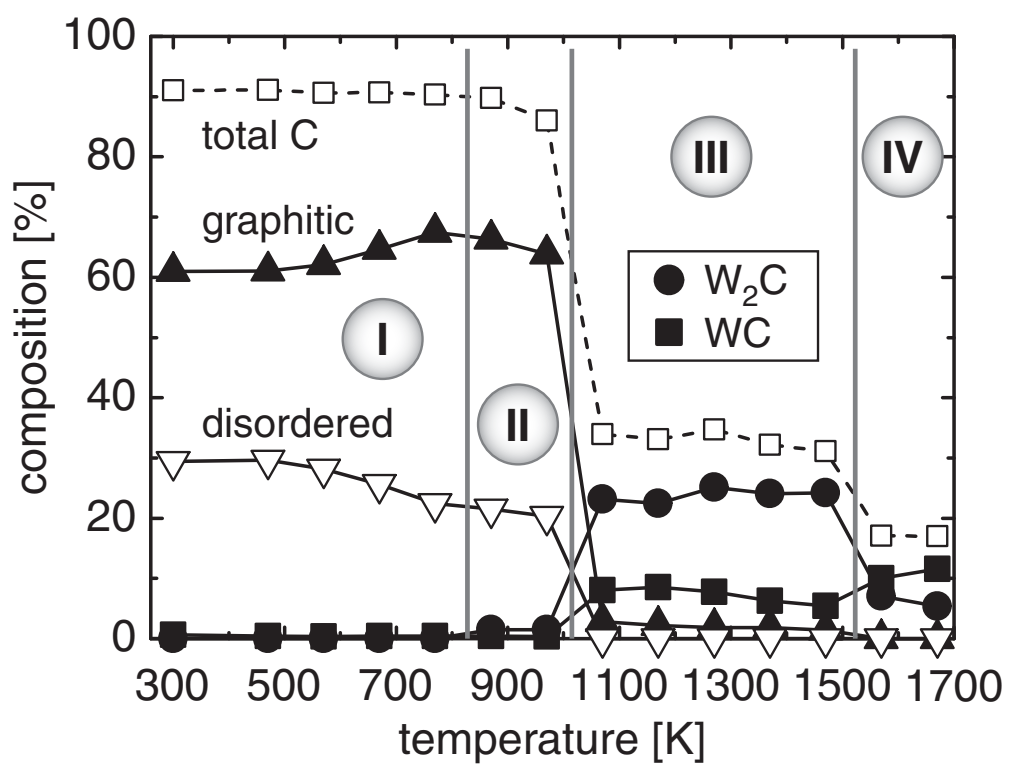

Fig. 1 


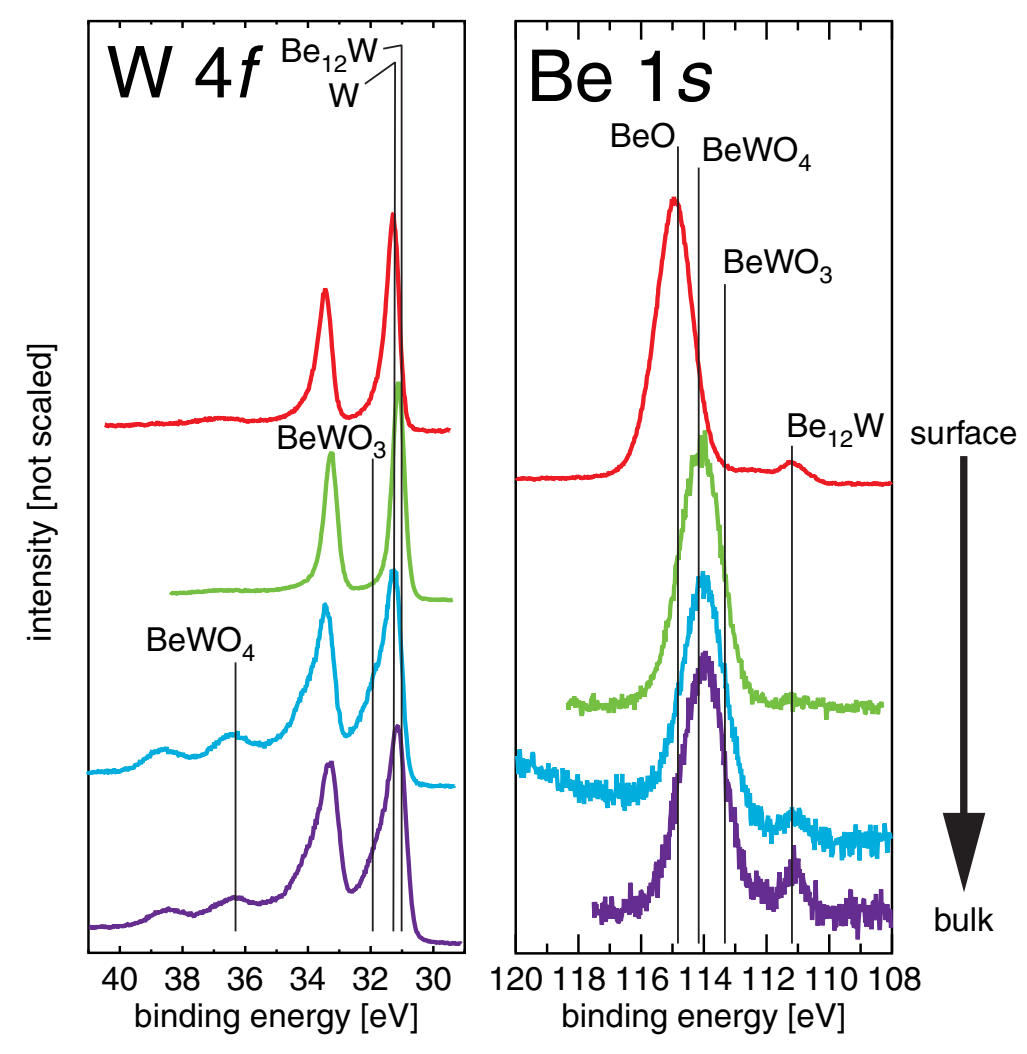

Fig. 2 

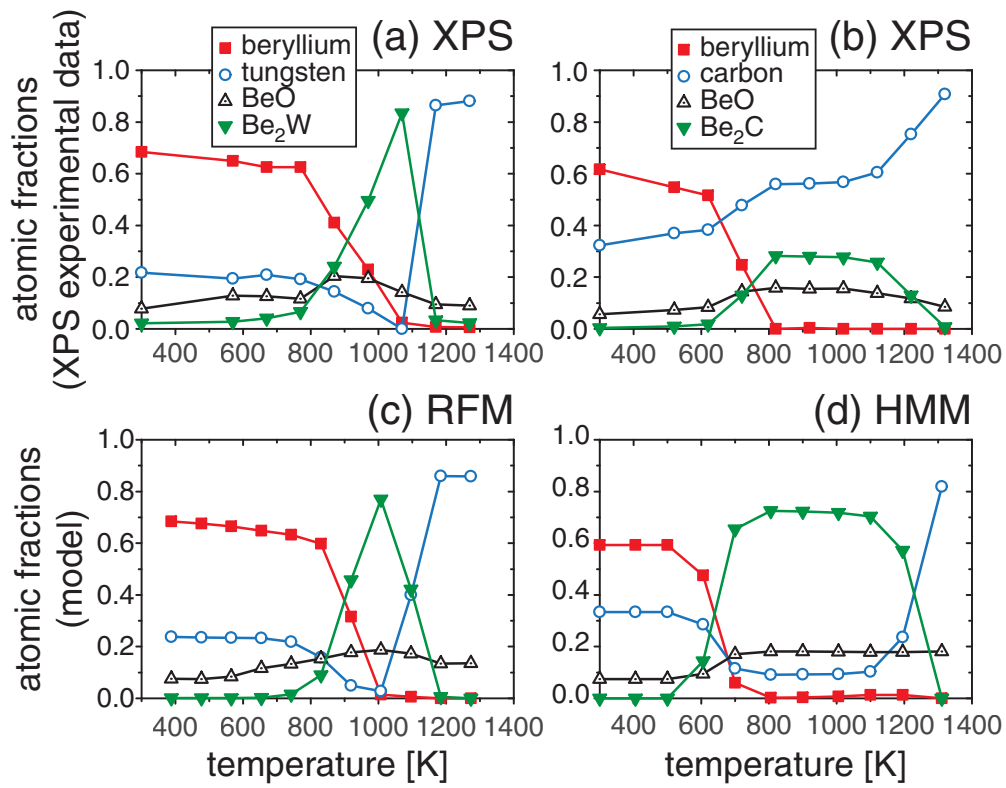

Fig. 3 